\title{
A simple proof of orientability in colored group field theory
}

Francesco Caravelli $i^{1,2,3}$

\begin{abstract}
Background: Group field theory is an emerging field at the boundary between Quantum Gravity, Statistical Mechanics and Quantum Field Theory and provides a path integral for the gluing of $\mathrm{n}$-simplices. Colored group field theory has been introduced in order to improve the renormalizability of the theory and associates colors to the faces of the simplices.

The theory of crystallizations is instead a field at the boundary between graph theory and combinatorial topology and deals with n-simplices as colored graphs. Several techniques have been introduced in order to study the topology of the pseudo-manifold associated to the colored graph.

Although of the similarity between colored group field theory and the theory of crystallizations, the connection between the two fields has never been made explicit.

Findings: In this short note we use results from the theory of crystallizations to prove that color in group field theories guarantees orientability of the piecewise linear pseudo-manifolds associated to each graph generated perturbatively.

Conclusions: Colored group field theories generate orientable pseudo-manifolds. The origin of orientability is the presence of two interaction vertices in the action of colored group field theories. In order to obtain the result, we made the connection between the theory of crystallizations and colored group field theory.
\end{abstract}

\section{Introduction}

There has been recently a growth of interest in group field theories Freidel (2005) and Oriti (2009) and there are many reasons for this to happen. Group field theories (GFT) are a generalization of matrix models to higher dimensions Brezin et al. (1978) and David (1985) and a generalization of tensor models as well Ambjorn et al. (1991). Moreover, GFT are known to generate the partition function of Spin Foams, thus having a direct relation with Loop Quantum Gravity Rovelli (2004).

It is known that matrix models have a topological expansion in which the genus, the only topological invariant needed to characterize orientable surfaces, plays the role of the parameter of this expansion. Roughly speaking a $n$ dimensional group field theory has a vertex associated to an $n$-simplex and a propagator which glues the $(n-1)$ simplices. Feynman diagrams of a $n$-dimensional group field theory can be interpreted as gluings of simplices

\footnotetext{
Correspondence: fcaravelli@perimeterinstitute.ca

1 University of Waterloo, Waterloo, Ontario N2L 3G1, Canada

2 Perimeter Institute for Theoretical Physics, Waterloo, Ontario N2L 2Y5, Canada
} Full list of author information is available at the end of the article and then have the interpretation of piecewise linear (PL) manifolds. A colored version of group field theory (cGFT) has been introduced recently Gurau (2009, 2010a) and Geloun et al. (2010a). One important reason to introduce color in the diagrams is that it is possible to have a better control over the perturbatively generated singularities of GFT. The challenge in these models is to obtain a topological expansion as in the 2-dimensional case Bonzom and Smerlak (2010); Freidel et al. (2009); Geloun et al. (2010b); Magnen et al. (2009). Remarkably, it has been shown Gurau (2010b) that spheres dominates the partition function in any dimension. In order to achieve this result, techniques from the theory of crystallizations have been used. In fact, colored $n$-graphs are well known in mathematics as gems: graph-encoded manifolds Pezzana (1974) and Lins (1995).

In this paper we use the results in this field of mathematics to show that the growth of interest in colored models is not unjustified: colored models generate orientable pseudomanifolds in any number of dimensions. Many of the theorems we will use were known for long time in the context of crystallization and here we report briefly these

\section{Springer}

(c) 2012 Caravelli; licensee Springer. This is an Open Access article distributed under the terms of the Creative Commons

Attribution License (http://creativecommons.org/licenses/by/2.0), which permits unrestricted use, distribution, and reproduction in any medium, provided the original work is properly cited. 
results. The outcome of this note is that the generation of pseudo-manifolds is due to the color, while the orientability in the colored versions of group field theory models is due to the presence of two different vertices (clockwise and anti-clockwise). In the following we will focus on the Boulatov model, but the result is more general because it relies only on the presence of vertices of opposite orientation in the perturbative expansion of the partition function. In fact, the result can be easily generalized to other types of theory with color and with a similar interaction structure, given the interpretation of the vertices as a simplicial complex.

Another point we would like to stress, is that the present paper establishes a dictionary between the mathematical field of crystallisation and colored graphs within the context of tensor field models. This dictionary and the results obtained in the theory of crystallisation allowed the simple proof of the result. We believe that these techniques could be very useful for the field. For instance, the proof of the $1 / N$ expansion used in Gurau (2010b) heavily relies on the dipole moves we will discuss later in the paper.

The paper is organized as follows: in section The colored boulatov model we recall the colored Boulatov model and its standard interpretation. In section A survey of graph-embedded manifolds results we review basic results in the field of 3-gems and crystallizations. We will use some of these results in section Orientability in cGFT to prove the orientability of simplicial complexes generated perturbatively by the colored Boulatov model. Conclusions follow.

\section{The colored boulatov model}

In this section we introduce the colored Boulatov model Boulatov (1992) and Gurau (2009). Let us consider a compact Lie group $H$, denote $h$ its elements, $e$ the unit element, and $\int d h$ the integral with respect to the Haar measure of the group.

In 3 dimensions we introduce two fields, $\bar{\psi}^{i}$ and $\psi^{i}$, $i=0,1,2,3$ be four couples of complex scalar (or Grassmann) fields over three copies of $G, \psi^{i}: G \times G \times G \rightarrow \mathbb{C}$. The index $i$ runs from $=0$ to $n+1$, where $n$ is the number of dimensions, and the $\psi$ and $\bar{\psi}$ are functions of $n$ copies of the group. In the fermionic version of the theory the indices $i$ can be seen as the dependence of the field from a (global) gauge group $S U(N)$, where $N=n+1$. We denote $\delta^{\Lambda}(h)$ the regularized delta function over $G$ with some cutoff $\Lambda$ such that $\delta^{\Lambda}(e)$ is finite, but diverges when $\Lambda$ goes to infinity. A feasible regularization is given, for instance for the group $G=S U(2)$, by

$$
\delta^{\Lambda}(h)=\sum_{j=0}^{\Lambda}(2 j+1) \chi^{j}(h) .
$$

where $\chi^{j}(h)$ is the character of $h$ in the representation $j$. The path integral for the colored Boulatov model over $G$ is:

$$
\begin{aligned}
Z(\lambda, \bar{\lambda}) & =e^{-F(\lambda, \bar{\lambda})} \\
& =\int \prod_{i=0}^{4} d \mu_{P}\left(\bar{\psi}^{i}, \psi^{i}\right) e^{-S^{i n t}\left(\bar{\psi}^{i}, \psi^{i}\right)},
\end{aligned}
$$

where the Gaussian measure $P$ is chosen such that:

$$
\int \prod_{i=0}^{4} d \mu_{P}\left(\bar{\psi}^{i}, \psi^{i}\right)=1,
$$

and:

$$
\begin{aligned}
& P_{h_{0} h_{1} h_{2} ; h_{0}^{\prime} h_{1}^{\prime} h_{2}^{\prime}}= \\
& =\int d \mu_{P}\left(\bar{\psi}^{i}, \psi^{i}\right) \bar{\psi}_{h_{0} h_{1} h_{2}}^{i} \psi_{h_{0}^{\prime} h_{1}^{\prime} h_{2}^{\prime}}^{i}= \\
& =\int d h \delta^{\Lambda}\left(h_{0} h\left(h_{0}^{\prime}\right)^{-1}\right) \delta^{\Lambda}\left(h_{1} h\left(h_{1}^{\prime}\right)^{-1}\right) \delta^{\Lambda}\left(h_{2} h\left(h_{2}^{\prime}\right)^{-1}\right),
\end{aligned}
$$

The fermionic colored model has two types interactions, a "clockwise" and an "anti-clockwise", and one is obtained from the other one by conjugation in the internal group color $\operatorname{SU}(N)$, where $N$ is 4 in 3 dimensions, one for each face of the 3-simplex ${ }^{\text {a }}$. For convenience we denote $\psi(h, p, q)=\psi_{h p q}$. Invariance under global rotations in the internal color group require at least two interactions:

$$
\begin{aligned}
S^{i n t}= & \frac{\lambda}{\sqrt{\delta^{\Lambda}(e)}} \int(d h)^{6} \psi^{0} \psi^{1} \psi^{2} \psi^{3} \\
& +\frac{\bar{\lambda}}{\sqrt{\delta^{\Lambda}(e)}} \int(d h)^{6} \bar{\psi}^{0} \bar{\psi}^{1} \bar{\psi}^{2} \bar{\psi}^{3}
\end{aligned}
$$

where we omitted the internal structure of the group elements of the fields $\psi^{i}$ and $\bar{\psi}^{i}$. In order to make the notation clearer (already the orientation of the colors is sufficient to distinguish the two vertices), we call "red" the vertex involving the $\psi$ 's and "black" the one involving the $\bar{\psi}$ 's. Thus any line coming out of a cGFT vertex has a color $i$.

The group elements $h_{i j}$ in eq. (3) are associated to the propagators (represented as solid lines), and glue two vertices with opposite orientation. The vertex can be seen as the dual of a tetrahedron and its lines represent the triangles which form the tetrahedron. Each propagators is decomposed into three parallel strands which are associated to the three arguments of the fields, i.e. the 1dimensional elements of the 1-skeleton of the tetrahedron which bound every face. These are associated to the edges of the tetrahedron. A colored line represents the gluing of two tetrahedra (of opposite orientations) along triangles of the same color as in Figure (1).

It is easy to understand that a cGFT graph can be seen either as a stranded graph (using the vertex and the propagators as depicted in Figure 2) or as a "colored graph" with 


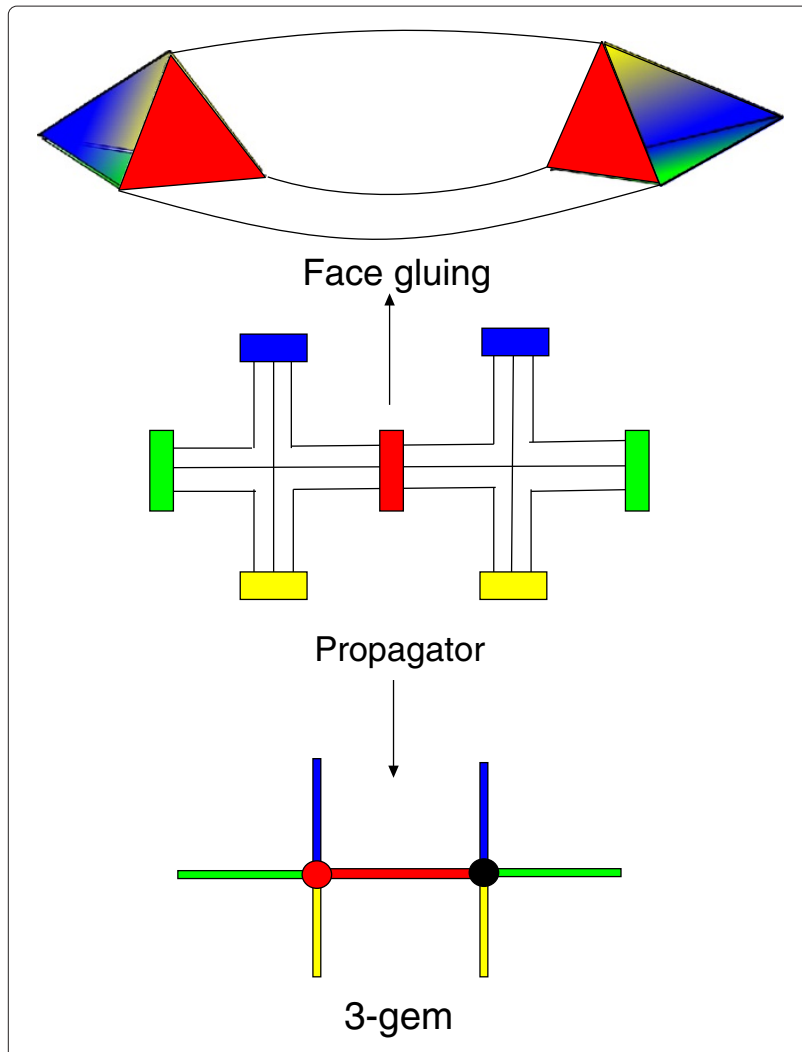

Figure 1 A gluing using a colored propagator.

(colored) solid lines, and two classes of oriented vertices. In this paper we consider only vacuum graphs, i.e. all the vertices of the graphs are 4-valent and we deal only with connected graphs (thus with the logarithm of the partition function (2)). The lines of a vacuum cGFT graph $\Gamma$ have two natural orientations given by the fact that only vertices of opposite orientations can be glued. It is easy to see that a vacuum cGFT graph must have the same number of black and red vertices. For any graph $\Gamma$, we denote $n$ as the number of vertices, $l$ as the lines of $\Gamma$, and we define as faces (not to be confused with the faces of the tetrahedron!), $\mathcal{F}_{\Gamma}$, as any closed strand in the Feynman graph of a GFT. Thus a generic vacuum Feynman amplitude of the theory can be written as:

$$
\mathcal{A}=\frac{(\lambda \bar{\lambda})^{\frac{n}{2}}}{\left[\delta^{N}(e)\right]^{\frac{n}{2}}} \int \prod_{l \in \Gamma} d h_{l} \prod_{f \in \mathscr{F}_{\Gamma}} \delta_{f}^{\Lambda}\left(\prod_{l_{0} \in f} h_{l_{0}}^{\sigma\left(l_{0}, f\right)}\right),
$$

where $l_{0}$ is a line associated to a face $f$ and $\sigma\left(l_{0}, f\right)$ is alternatively +1 or -1 depending on the orientation. In the following we will assume that an orientation is fixed. Because of the properties of $\delta^{\prime} s$ the orientation does not affect the amplitude. To each colored graph associated to an amplitude of the colored Boulatov model it is possible to associate bubbles by removing all the edges of one color. We call $\mathcal{B}_{i_{1}, \cdots, i_{k}}$ the set of $k$-bubbles associated to

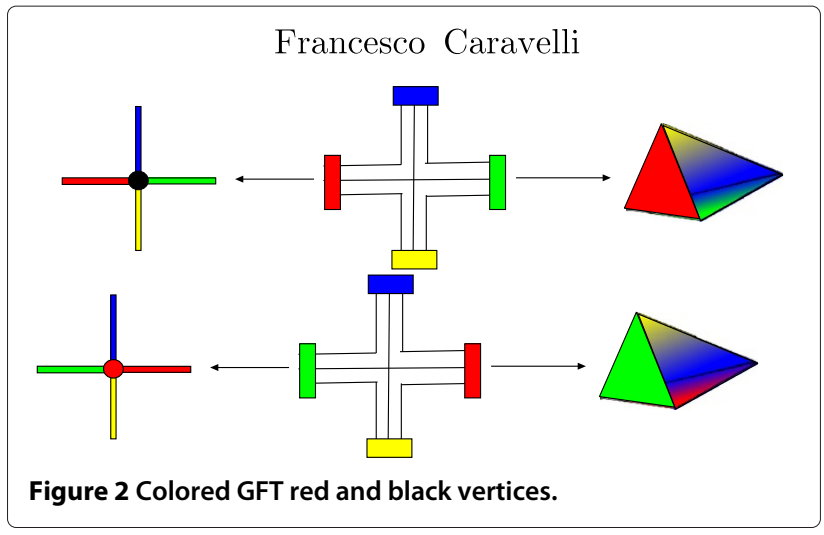

the deletion of $n-k$ colors. In 3-dimensions, for instance, 3 -bubbles have 3-colors (surfaces), 2-bubbles have 2 colors (lines) and so on and so forth. Bubbles play a special role in the theory, since they discriminate manifold from pseudo-manifolds (see next section for the same result in the theory of 3-gems).

\section{A survey of graph-embedded manifolds results}

In this section we review some basic results in the field of 3-gems and make a dictionary between the two literatures, as colored group field theory can gain much from the results obtained in all the years of research in such field.

Let $\Gamma$ be a finite, edge-colored graph, parallel edges allowed. A $k$-residue of $\Gamma, k \in \mathbf{N}$ is a connected component of subgraph of $\Gamma$ induced by $k$ color classes (this is what in colored group field theory are called bubbles). These graphs represent a piecewice linear manifold in the following sense (a pseudo-complex) Ferri et al. (1986). A $n$-regular $n$-colored graph is an edge-colored graph which has a nodes of degree $n$. To a couple $(\Gamma, \gamma)_{n+1}$ there is an associated pseudo-complex $K(\Gamma)$ given by the following construction. Take an $n$-simplex $\sigma^{n}$ for each $V(\Gamma)$ and label its vertices $\Delta_{n}$. If $x, y$ in $V(\Gamma)$ are joined by an edge, then attach the $(n-1)$-faces of their associated simplices. This is the same interpretation given to attaching faces of $n$-simplices in a $n$-dimensional group field theory. We denote $|\Gamma|$ the pseudo-complex associated with the colored graph $\Gamma$.

Lemma 1. For any PL n-manifold $\mathcal{M}$ there exist a $(n+1)$ graph $\Gamma$ such that $|\Gamma| \simeq \mathcal{M}$.

We now restrict to the case of 3-dimensions and list some of the basic results Lins (1995). Let $\Gamma$ be a 4-edge-colored 4-graph and denote by $v, e, b, t$ respectively the number of vertices (0-residues), edges (1-residues), 2-residues and 3residues.

Definition 1. A 3-gem (a 3 graph-embedded manifold) is a 4-regular properly edge-colored graph such that

$$
v+t=b
$$


A 4-regular properly edge-colored graph for which (5) does not apply is called 3-gepm (a 3 graph-embedded pseudomanifold).

Lemma 2. A necessary and sufficient condition for the graph $(\Gamma, \gamma)_{4}$ to represent a manifold, is to meet the relation between its 2- and 3-residues (read as it 2- and 3colored bubbles) and the number of vertices (read as the perturbative order) $v+t=b$. This Lemma clarifies the reason why 3-gems have to satisfy the relation (5). Let now introduce few definitions which will turn useful later Ferri et al. (1986):

Definition 2. A triball is a connected, cubic, 3-edgecolored graph $\Gamma_{3} \subset \Gamma$ such that its Euler characteristic is the one of the 2-sphere. Thus we have the relation between its 2-residues $b_{\Gamma_{3}}$ and the vertices: $2 b_{\Gamma_{3}}-v=4 . A n$ important fact is the following:

Lemma 3. A graph $(\Gamma, \gamma)_{4}$ is a 3-gem iff each of its 3residue is a triball. Thus, the condition that graphs have to satisfy in order to be 3-gems is a condition on the topology of its 3-residues. We now discuss crystallizations of 3-gems [Ferri and Gagliardi (1982); Lins (1995); Lins and Mandel (1985)]. Let first introduce the fusion process. Let be $\mathcal{B}_{i j k}$ and $\mathcal{B}^{\prime}{ }_{i j k}$ two different 3-residues separated by a unique color which, by construction, is different from the color $i$, $j$, $k$. We call 1 - dipole this edge connecting the two 3residues. The generalization to $k$-dipoles which connect $(n-k)$-residues is obvious. We call fusion the process of contraction of two vertices through the first two combinatorial moves depicted in Figure 3. Each cancellation of a 1-dipole has the effect of decreasing by one the number of $i$-residues, where $i$ is the color of the edge which defines the 1 -dipole, not changing the number of $j$-residues, for $j \neq i$. Thus by a succession of 1-dipole cancellation we obtain a 3-gem with 4 triballs. Such a 3-gem is said to be contracted and is called a crystallization for the associated 3-manifold. It is a fact that any closed 3-manifolds has a crystallization, and two closed 3-manifolds are related by a homeomorphism if and only if they are related by creation or contraction of 1- and 2-dipoles with the fusion rules; in this case, the two 3-manifolds are said to be equivalent or homeomorphic. Thus it is easy to understand that the fusion rules are the combinatorial equivalent of homeomorphisms. Let now discuss crystallization for generic colored $(n+1)$-graphs. The following results hold:

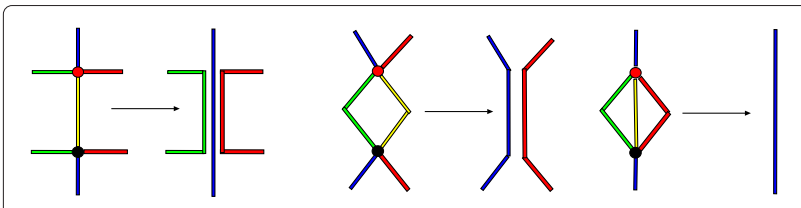

Figure 3 Fusion moves on a 4-regular 4-edge colored graph of 1-, 2- and 3-dipoles respectively.
Theorem 1. For every $P L n$-manifold $\mathcal{M}$ there exist a crystallization.

Theorem 2. Two n-graphs $\left|\Gamma_{1}\right|$ and $\left|\Gamma_{2}\right|$ are crystallizations of the same manifold $\mathcal{M}$ if one is converted into the other by:

a) Adding or removing a non-degenerate $m$-dipole with $n-1>m>1$;

b) Adding a 1-dipole and deleting another 1-dipole.

A general theorem on the orientability of n-graphs holds:

Theorem 3. (Orientability) Let $(\Gamma, \gamma)_{n+1}$ be any crystallization of an $n$-manifold $\mathcal{M}$. Then $\mathcal{M}$ is orientable iff $\Gamma$ is bipartite. These theorems are fundamental in order to have a clear geometrical understanding of graphs generated by a colored group field theory i.e. that to vertices there are associated n-simplices. This interpretation will be used in the next section, in which the main result of the paper is presented.

\section{Orientability in CGFT}

In this section we prove a Lemma on the orientability of PL manifolds associated to graphs generated by the colored Boulatov model. Orientability of a manifold is a requirement if we want to construct a spin bundle. In 4-dimensions, for instance, the requirement to have a global spin bundle is to have a vanishing first and second Stiefel-Whitney class. While the second can be neglected by constructing local spin bundle and then gluing the charts, the vanishing of the first is a strict requirement and is equivalent to ask the orientability of the manifold. Another important fact is that orientability restricts enormously the class of 3-manifolds which could be generated. As an example, in 2-dimensions the most general decomposition is given by connected sum of spheres, torii and projective planes. Orientability excludes the connected sum of projective planes, which allows the expansion in the ordinary genus we are used to.

Lemma 4. (3-dimensions) Let $\Gamma$ be a connected vacuum finite graph generated by the colored Boulatov model. Let $\mathcal{B}_{i j k}$ and $\mathcal{B}_{i j}$ be the set of 3-and 2-bubbles of $\Gamma$ respectively. Then the pseudo-manifolds associated to $\Gamma$ is an oriented pseudo-manifold. Moreover, $|\Gamma|$ represents a closed and orientable 3-manifold iff

$$
V+\operatorname{Card}\left\{\mathcal{B}_{i j k}\right\}=\operatorname{Card}\left\{\mathcal{B}_{i j}\right\}
$$

Proof. This lemma follows directly from the properties of graphs generated by the colored Boulatov model and its interpretation, which is the same of the simplicial construction of 3-gepms. By Lemma 1 the graph generated is a manifold if and only if the condition (6) is met. Since the graph is finite, the manifold is also closed. Thus what we 
have to show is that they are orientable. By the theorem on the orientability the 3-gem represents an orientable manifold if and only if the crystallization graph is bipartite. First we note that the graphs generated by colored group field theory are bipartite. Let $A$ and $B$ be the set of clockwise and anti-clockwise vertices of $\Gamma$ respectively. Since by construction a clockwise vertex has to be contracted with an anticlockwise, then all the edges are between the set $A$ and the set $B$ and none is within the sets, thus the graph is bipartite. Now we have to show that its contraction is still bipartite. However, this fact is trivial because any of the moves in Figure 3 keeps the bipartiteness of the original graph, thus in particular the fusion of a 1 - dipole. Moreover, since the graph is finite, the crystallization is reached in a finite number of moves.

The orientability part of this Lemma can be generalized to higher dimensions. The construction given in the third section of this note ensures that to each $n$-dimensional pseudo-complex there is at least a colored $(n+1)$-graph which is homehomorphic to it. It is then easy to see why colored group field theories generates only orientable pseudo-manifolds in any number of dimensions; we state it as a Lemma, even if it clearly follows from the construction given in Pezzana (1974) of $n$-edge-colored graphs in any number of dimensions, while orientability comes from a generalization to m-dipoles (as in Theorem 2) of the previous proof and the fact that there are two types of vertices: This means that, at any finite order, the connected vacuum graphs generated by the partition function of a colored group field theory are associated with closed and orientable PL pseudo-manifolds.

\section{Conclusions}

In this short paper we have used results in the field of 3 -gems to prove that all the graphs generated by the colored Boulatov model are related to orientable pseudomanifolds. In order to prove it we used new tools which could turn to be very useful in the context of group field theory, more specifically in the colored version of it. In fact, color is a fundamental ingredient in all we said. It should be said that what proved here is not an unexpected result Oriti and Gurau (2010). The fact that an orientation for the faces can be chosen with ease was a hint of what proved here. Indeed, as far as the author is concerned, this is the first rigorous proof appeared so far.

\section{Endnote}

${ }^{a}$ It should be mentioned that also in the bosonic version Geloun et al. (2010a) there is a clockwise and anti-clockwise interaction. In that case the the types of interactions are motivated by the fact that its introduction has a nice combinatorial definition of homology Gurau (2010a).

\section{Competing interests}

The authors declare that they have no competing interests.

\section{Acknowledgements}

We would like to thank Razvan Gurau for several discussions on the topic of group field theory. Also, we would like to thank Daniele Oriti for advices on the presentation of this result and Lorenzo Sindoni for reading carefully the manuscript. Research at Perimeter Institute for Theoretical Physics is supported in part by the Government of Canada through NSERC and by the Province of Ontario through MRI.

\section{Author details}

${ }^{1}$ University of Waterloo, Waterloo, Ontario N2L 3G1, Canada. ${ }^{2}$ Perimeter Institute for Theoretical Physics, Waterloo, Ontario N2L 2Y5, Canada. ${ }^{3}$ Max Planck Institute for Gravitational Physics (Albert Einstein Institute), Am Mühlenberg 1, D-14476 Golm, Germany.

Received: 16 April 2012 Accepted: 27 April 2012

Published: 5 July 2012

\section{References}

Ambjorn J, Durhuus B, Jonsson T (1991) Three-Dimensional Simplicial Quantum Gravity And Generalized Matrix Models. Mod Phys Lett A 6: 1133 Bonzom V, Smerlak M (2010) Lett Math Phys 93: 295. [arXiv:1004.5196 [gr-qc]]

Brezin E, Itzykson C, Parisi G, Zuber JB (1978) Planar Diagrams. Commun Math Phys 59: 35

Boulatov DV (1992). A Model of three-dimensional lattice gravity. Mod Phys Lett A 7, 1629. [arXiv:hep-th/9202074]

David F (1985) A Model Of Random Surfaces With Nontrivial Critical Behavior. Nucl Phys B 257: 543

Ferri, M, Gagliardi C (1982) Crystallisation moves. Pac J Mathematics 100(1) Ferri M, Gagliardi C, Grasselli L (1986) A graph-theoretical representation of PL-manifolds - A survey on crystallizations. Aequationes Mathematicae 31: $121-141$

Freidel L (2005) Group field theory: An overview. Int J Theor Phys 44: 1769. [arXiv:hep-th/0505016]

Freidel L, Gurau R, Oriti D (2009) Group field theory renormalization - the 3d case: power counting of divergences. Phys Rev D 80: 044007. [arXiv:0905.3772 [hep-th]]

Geloun J B, Magnen J, Rivasseau V (2010a) Bosonic Colored Group Field Theory. [arXiv:0911.1719 [hep-th]]

Geloun J B, Krajewski T, Magnen J, Rivasseau V (2010b) Linearized Group Field Theory and Power Counting Theorems. Class Quant Grav 27: 155012. [arXiv:1002.3592 [hep-th]]

Gurau R (2009) Colored Group Field Theory. [arXiv:0907.2582 [hep-th]]

Gurau R (2010a) Topological Graph Polynomials in Colored Group Field Theory. Annales Henri Poincare 11: 565. [arXiv:0911.1945 [hep-th]]

Gurau R (2010b) The 1/N expansion of colored tensor models. [arXiv:1011.2726v2 [gr-qc]]

Lins S (1995). Gems, Computers and Attractors for 3-Manifolds, (Series on Knots and Everything, Vol 5). ISBN: $9810219075 /$ ISBN-13: 9789810219079

Lins S, Mandel A (1985) "Graph-encoded 3-manifolds". Discrete Mathematics 57: $261-284$

Magnen J, Noui K, Rivasseau V, Smerlak M (2009) Scaling behaviour of three-dimensional group field theory. Class Quant Grav 26: 185012 [arXiv:0906.5477 [hep-th]]

Oriti D (2009). The group field theory approach to quantum gravity: some recent results. [arXiv:0912.2441 [hep-th]]

Oriti D, Gurau R (2010) Private communications.

Pezzana M (1974) Sulla struttura topologica delle varietà compatte. Atti Sem Mat Fis Univ Modena 23: 269-277

Rovelli C (2004). Quantum Gravity, Cambridge University Press, Cambridge; Perez, A "Introduction to Loop Quantum Gravity and Spin Foams." [arXiv:gr-qc/0409061]

doi:10.1186/2193-1801-1-6

Cite this article as: Caravelli: A simple proof of orientability in colored group field theory. SpringerPlus 2012 1:6. 